\title{
Fast Frequency-Hopping Dynamic Multiple-Access for Cognitive Radios: Suboptimum Noncoherent Maximum-Likelihood Multiuser Detection
}

\author{
Shuo Zhang ${ }^{\# 1}$, Lie-Liang Yang ${ }^{* 2}$, Youguang Zhang \#3 \\ \# School of Electronic and Information Engineering \\ Beihang University, Beijing 100191, China \\ ${ }^{1}$ sz09veecs.soton.ac.uk \\ ${ }^{3}$ zhangyouguang@vip.sina.com \\ * School of Electonics and Computer Science \\ University of Southampton, SO17 1BJ, United Kingdom \\ ${ }^{2}$ lly@ecs.soton.ac.uk
}

\begin{abstract}
We propose a novel dynamic multiple-access (DMA) scheme for application in cognitive radios (CRs). This DMA scheme is implemented by fast frequency hopping with $M$ ary frequency-shift keying (FFH/MFSK) associated with suboptimum noncoherent maximum-likelihood multiuser detection (ML-MUD). In our studies, we assume that the primary users (PUs) and the cognitive radio users (CRUs) are operated in the interweave paradigm. The PUs activate to communicate according to a Poisson process and the duration of an activation obeys exponential distribution. The bit error rate (BER) and throughput performance of the dynamic FFH/MFSK systems are investigated by simulations, when assuming communications over Rayleigh fading channels. Our studies and simulation results demonstrate that the dynamic FFH/MFSK with suboptimum noncoherent ML-MUD constitutes one of the promising candidates for DMA in CRs. It has low-complexity and employs highflexibility for DMA and seamless transition between different frequency bands.
\end{abstract}

\section{INTRODUCTION}

The increasing demand for high-speed wireless multimedia services makes radio spectrum one of the most scarce and valuable resources, the use of which has been regulated by governments and usually limited only for licensed users. However, the practical measurement shows that most of the licensed spectrum is underutilised and low-efficiency $[1,2]$. The studies in recent years demonstrate that cognitive radios (CRs) [3] may have the potential to increase the efficiency of the radio spectrum through introducing opportunistic access of the licensed frequency bands that are underutilised [2-4]. Furthermore, it has been shown that the main techniques in CRs include [4]: (1) the spectrum sensing, which determines available spectrum holes [5] for CRUs and detects the presence of PUs, (2) the spectrum management, which motivates to make the spectral-efficiency as high as possible, (3) the spectrum sharing for coordinating the CRUs to access available spectrum and, (4) the spectrum mobility, which concerns seamless transition from one spectrum to another.

In this paper, we focus on the issue of spectrum sharing and consider the DMA in CRs through noncoherent FFH/MFSK schemes $[12,13]$. In order to investigate the performance of the dynamic FFH/MFSK system, we assume that the PUs and CRUs are operated in the interweave paradigm [10], where the CRUs can only communicate opportunistically on the frequency bands not used by the PUs, and must stop communication, once there are PUs appearing on these bands. We assume that the whole frequency spectrum accessible by the CRUs is divided into a number of frequency bands. Once a PU activates, it occupies one to several frequency bands. We assume that the PUs activate according to a Poisson process and the duration of activation of a PU obeys exponential distribution. Therefore, the frequency bands possible for the CR system is dynamic and is utilised by the dynamic FFH/MFSK scheme, which varies the set of FFH frequencies according to the frequency bands available. In this paper, a noncoherent suboptimum ML-MUD is proposed for detection of the dynamic FFH/MFSK signals. Both the BER and throughput performance of the dynamic FFH/MFSK systems are investigated by simulations, when communication over Rayleigh fading channels is assumed. Our studies and simulation results show that the dynamic FFH/MFSK is a highefficiency scheme for DMA in CRs. It has low implementation complexity owing to using noncoherent detection. With the aid of the FFH techniques, it is very easy to achieve seamless spectrum transition. Furthermore, assisted by the suboptimum ML-MUD, the dynamic FFH/MFSK system is capable of achieving very promising error performance and throughput performance even in Rayleigh fading channels, owing to the characteristics that the FFH scheme can naturally make use of the frequency-selective diversity [12].

Note that, since the proposal of CRs, DMA has attracted a lot of attention in research, as evidenced by, e.g., [4, 6-8]. However, the DMA schemes considered so far for CRs use mainly coherent detection depending on ideal channel state information (CSI), which is hard to acquire in the dynamic CR communication environments. Furthermore, the dependence on real-time CSI imposes specific requirements on signal 
design and requires extra bandwidth for channel estimation, which unavoidably decrease the spectrum-efficiency of the CR systems.

\section{SyStem Modelling}

In our considered systems, there are two types of users without cross-type cooperation. The first are the PUs that are licensed to use the spectrum bands. The second are the CRUs or secondary users, which are not pre-assigned wireless spectrum bands. In this paper, we assume that the PUs and CRUs are operated under the interweave paradigm [10], where the CRUs can only communicate opportunistically on the unused spectrums referred to as 'spectrum holes', without affecting the PUs' communication and reducing the qualityof-service (QoS) of the primary communications systems. In other words, the CRUs must stop communication on a spectrum band, once the PUs licensed to use the band appear.

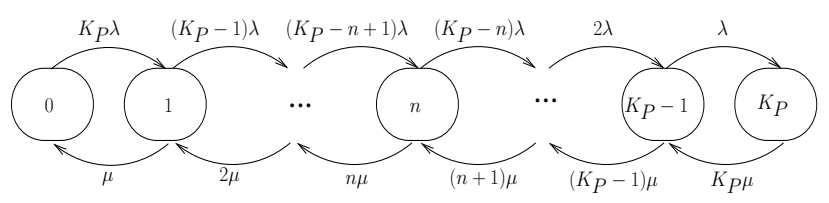

Fig. 1. State transition diagram for the arrival process of PUs.

For the sake of carrying out the related investigation, we assume that the total spectrum accessible by the CR users is divided into $C$ channels, each of which has a fixed and equal bandwidth that is enough for accommodating a MFSK tone. It is assumed that, in the primary communications network, these $C$ channels have been allocated to support $K_{P}$ PUs, which are activated according to the $M / M / K_{P} / K_{P} / K_{P}$ queueing model [9], which is depicted in Fig. 1. According to the $M / M / K_{P} / K_{P} / K_{P}$ queueing model, the number of active PUs follows the Poisson distribution associated with an intensity of $\lambda$ (arrival rate), the service time obeys the negative exponential distribution with an average service time of $1 / \mu$, while the number of parallel service windows, system capacity and the total number of customers are the same value of $K_{P}[9,11]$. Furthermore, let $c_{k}$ be the number of channels occupied by the $k$ th PU once it communicates. We assume that $\sum_{k=1}^{K_{P}} c_{k} \leq C$.

When the system reaches its steady state, according to the queueing theory [11], we can show that the probability that there are $n$ active PUs is

$$
P_{n}= \begin{cases}\frac{1}{K_{P} ! \sum_{i=0}^{K_{P}} \frac{1}{\left(K_{P}-i\right) ! i !}\left(\frac{\lambda}{\mu}\right)^{i}}, & n=0 \\ \frac{K_{P} !}{\left(K_{P}-n\right) ! n !}\left(\frac{\lambda}{\mu}\right)^{n} P_{0}, & 1 \leq n \leq K_{P}\end{cases}
$$

Furthermore, the number of bands available for the CRUs is given by

$$
\bar{C}=C-\sum_{k=1}^{K_{P}} \eta(k) c_{k}
$$

where $\eta(k)$ is a function defined as

$$
\eta(k)= \begin{cases}1, & \text { when the } k \text { th PU is ON } \\ 0, & \text { when the } k \text { th PU is OFF }\end{cases}
$$

We assume that an activated PU is allocated the channels chosen uniformly from the unused channels. In this case, it can be readily shown that the channels available for the CRUs constitute a Poisson process with its parameters that can be readily derived from the corresponding PUs' Poisson process. In this paper, we assume for simplicity that $C_{P}=c_{1}=c_{2}=$ $\ldots=c_{K_{P}}$. Then, when there are $n$ active PUs, the number of channels available for the CRUs is

$$
\bar{C}=C-n C_{P}, n=0,1, \ldots, K_{P}
$$

For the CRUs, we assume that they have the same priority to utilise the $\bar{C}$ bands, which is achieved by a noncoherent FFH/MFSK communication scheme considered in the next section.

\section{DYNAMIC NONCOHERENT FFH/MFSK SCHEME}

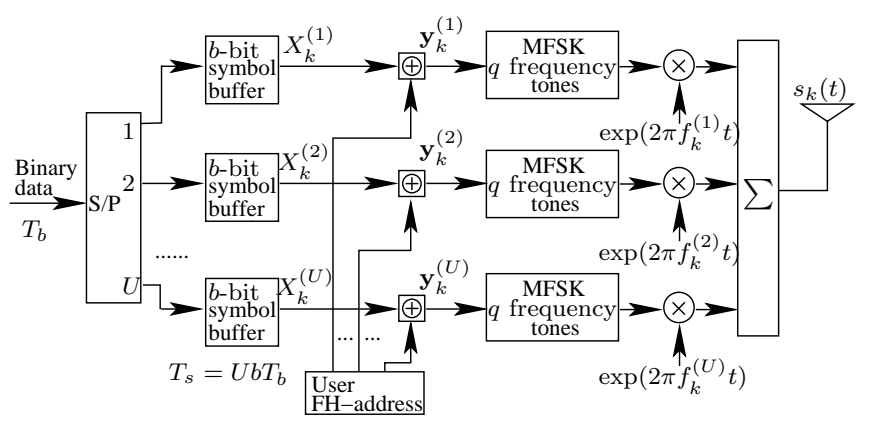

Fig. 2. The $k$ th user's transmitter schematic for the FFH/MFSK.

The general transmitter schematic diagram for the $k$ th CRU is shown in Fig. 2. It consists of $U$ sub-streams for the purpose to attain various data rates, each sub-stream carries out both FFH and MFSK operations. In more detail, as shown in Fig. 2, the input binary bits having a period $T_{b}$ and a rate $R_{b}$ is serial-to-parallel ( $\left.\mathrm{S} / \mathrm{P}\right)$ converted to $U$ parallel substreams. During a MFSK symbol interval of $T_{s}$ seconds, each sub-stream transmits $b=\log _{2} M$ bits representing a MFSK symbol. Hence, we have $T_{s}=U b T_{b}$. In order to implement the FFH, the symbol duration $T_{s}$ is divided into $L=T_{s} / T_{h}$ number of time slots of duration $T_{h}$, which represents the FH dwell time. For each of the sub-streams, a frequency is activated from a set of $q(q \geq M)$ frequencies according to the $k$ th CRU's FH address and the value of the MFSK symbol to be transmitted. The frequency hops from one to another per $T_{h}$ seconds.

According to the above description and Fig. 2, we can know that the total number of frequencies required by the general FFH/MFSK system is $Q=U q$. However, in our forthcoming discourse, we assume for simplicity that $U=1$ and, hence, $Q=q$. Note that, this simplification does not 
loss any generality, if we assume that the $U$ sub-streams are operated on $U$ orthogonal frequency bands.

Let the FH address code of CRU $k$ be expressed by $\boldsymbol{a}_{k}=\left[a_{k}(0), a_{k}(1), \ldots, a_{k}(L-1)\right]$ and $X_{k}$ be the symbol to be transmitted by CRU $k$. Then, as shown in Fig. $2, X_{k}$ is first signatured by $\boldsymbol{a}_{k}$, expressed as

$$
\boldsymbol{y}_{k}=\left[y_{k}(0), y_{k}(1), \ldots, y_{k}(L-1)\right]=X_{k} \cdot \mathbf{1} \oplus \boldsymbol{a}_{k}
$$

where 1 represents an all-one vector of length $L$ and $\oplus$ denotes the modulo- $Q$ addition operation. After the signaturing processing of (5), finally, each of the elements of $\boldsymbol{y}_{k}$ activates one from the $Q$ frequencies based on the MFSK principles and this activated frequency is transmitted for one time slot duration of $T_{h}$ seconds. Specifically, the transmitted signal for the MFSK symbol $X_{k}$ during $i T_{s} \leq t \leq(i+1) T_{s}$ can be expressed as

$s_{k}(t)=\sum_{l=0}^{L-1} \sqrt{2 P} \psi_{T_{h}}\left(t-i T_{s}-l T_{h}\right) \exp \left(2 \pi\left[f_{c}+f_{l}^{(k)}\right] t+\varphi_{l}^{(k)}\right)$

where $P$ denotes the transmission power per dimension, $\psi_{T_{h}}(t)$ is the normalised pulse waveform of duration $T_{h}, f_{l}^{(k)}$ is the frequency tone determined by $a_{k}(l)$ and $X_{k}, f_{c}$ is the carrier frequency, while $\varphi_{l}^{(k)}$ is the initial phase introduced by the MFSK and carrier modulation.

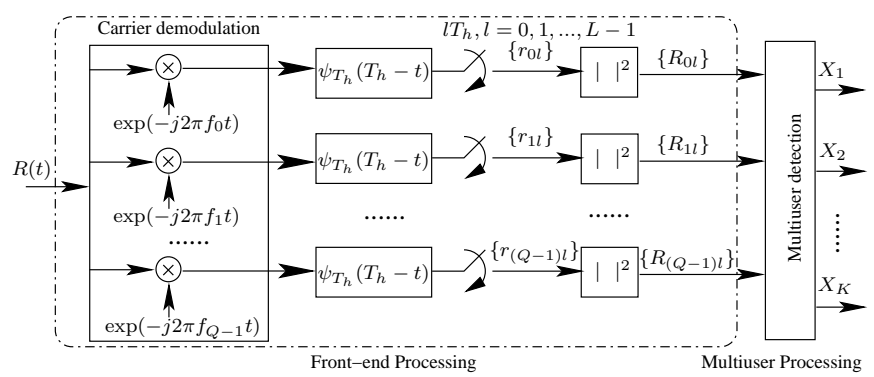

Fig. 3. Receiver schematic block diagram of the FFH/MFSK system.

We assume that there are $K$ CRUs communicating with a CR base-station (CRBS). The receiver schematic block diagram at the CRBS is shown in Fig. 3, which employs the posterior noncoherent MUD [12], as detailed later. As shown in Fig. 3, the receiver is divided into two sub-blocks: frontend processing and multiuser processing (MUP). We assume for simplicity that the CRU's signals are ideally synchronised and that their received power at the CRBS is the same. Consequently, the received complex low-pass equivalent signal at the CRBS can be expressed as

$$
R(t)=\sum_{k=1}^{K} \sqrt{2 P} \sum_{l=0}^{L-1} h_{l}^{(k)} \psi_{T_{h}}\left(t-l T_{h}\right) \exp \left(2 \pi f_{l}^{(k)} t\right)+N(t)
$$

where $h_{l}^{(k)}$ represents the fading gain of the channel with respect to the frequency $f_{l}^{(k)}$ of the $k$ th CRU and $N(t)$ is the complex valued low-pass equivalent additive white Gaussian noise (AWGN) with zero mean and single-sided power spectral density of $N_{0}$ per dimension. As shown in Fig. 3, the frontend processing sub-block is a typical noncoherent MFSK detector [13] excluding the decision-making device. It can be readily shown that, after some normalisation, the observation samples input to the MUP of Fig. 3 can be expressed as

$$
\begin{aligned}
R_{m l}= & \left|r_{m l}\right|^{2}=\left|\sum_{k=1}^{K} h_{m}^{(k)} \delta\left[y_{k}(l), m\right]+n_{m l}\right|^{2} \\
& m=0,1, \ldots, Q-1 ; \quad l=0,1, \ldots, L-1
\end{aligned}
$$

where $\delta[x, y]$ is the Dirac delta function, which is defined as $\delta[x, x]=1$ and $\delta[x, y]=0$ when $x \neq y, n_{m l}$ is a complex Gaussian noise sample distributed with mean zero and a variance $\sigma^{2}=L N_{0} / E_{s}$, where $E_{s}=P T_{s}$ denotes the energy per MFSK symbol.
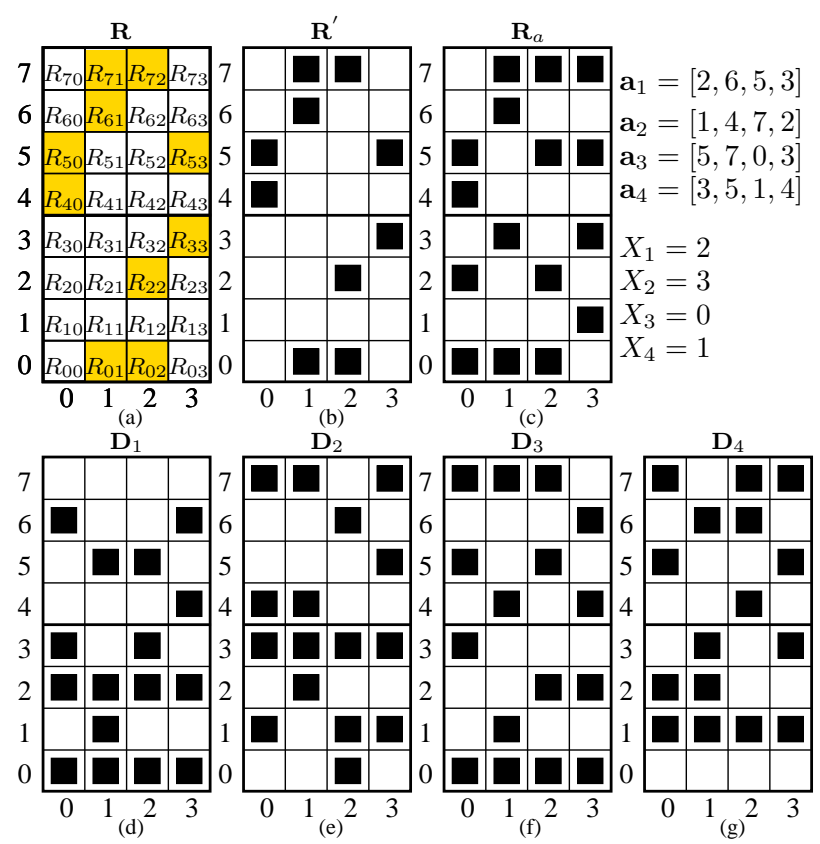

Fig. 4. TF matrices related to posterior noncoherent multiuser detection, when $M=4, Q=8, L=4$ : (a) soft-decision observations; (b) harddecision observations; (c) accompanying TF matrix $\boldsymbol{R}_{a}$; (d) (e) (f) (g) the $\mathrm{TF}$ matrices formed after the frequency-de-hopping operations using the $\mathrm{FH}$ addresses of CRUs 1,2,3 and 4 for soft-decision suboptimum ML-MUD.

In Fig. 3, the MUP is implemented by the posterior noncoherent MUD [12], which is based either on soft-decision or on hard-decision observations. Specifically, let us use $\left\{R_{m l}\right\}$ to construct a $(Q \times L)$ time-frequency (TF) matrix $\boldsymbol{R}=\left[R_{m l}\right]$, where the $Q$ rows are in the frequency (F)-domain explained by the $Q$ number of $\mathrm{FH}$ frequencies, while the columns are in the time (T)-domain determined by the $L$ time slots per symbol duration. In the context of the posterior noncoherent MUDs using soft-decision observations, the TF matrix is shown in Fig. 4(a), where the $(m, l)$ th element of the TF matrix is simply $R_{m l}$ and has analog value. For the posterior noncoherent MUDs using hard-decision observations, the TF 
matrix is shown in Fig. 4(b), where the elements of the TF matrix are binary, representing two states ' 1 ' (or 'marked') when $R_{m l} \geq \nu$, and ' 0 ' (or 'empty') when $R_{m l}<\nu$, where $\nu$ is a preset threshold for hard-decision making.

Let assume that the transmitted MFSK symbols by the $K$ CRUs are collected into a vector expressed as $\boldsymbol{s}=$ $\left[X_{1}, X_{2}, \ldots, X_{K}\right]^{T}$. Given the transmitted symbol vector $\boldsymbol{s}$ and a set of FH addresses of the $K$ CRUs, we express the conditional probability density function (PDF) of the received TF matrix $\boldsymbol{R}$ as $f\left(\boldsymbol{R} \mid \boldsymbol{s},\left\{\boldsymbol{a}_{k}\right\}\right)$. Then, the transmitted symbol vector $s$ can be detected by the optimum noncoherent MLMUD as

$$
\hat{\boldsymbol{s}}=\arg \max _{\boldsymbol{s} \in \mathcal{X}^{K}}\left\{f\left(\boldsymbol{R} \mid \boldsymbol{s},\left\{\boldsymbol{a}_{k}\right\}\right)\right\}
$$

where $\mathcal{X}$ is the signal set containing the $M$ possible MFSK symbols. We assume that the $Q L$ entries in the TF matrix $\boldsymbol{R}$ are independent. Then, the optimum noncoherent ML-MUD of (9) can be expressed as

$$
\hat{\boldsymbol{s}}=\arg \max _{\left\{X_{k} \in \mathcal{X}\right\}_{k=1}^{K}}\left\{\prod_{m=0}^{Q-1} \prod_{l=0}^{L-1} f\left(R_{m l} \mid \boldsymbol{s},\left\{\boldsymbol{a}_{k}\right\}\right)\right\}
$$

In this paper, we assume that the FFH/MFSK signals are transmitted over Rayleigh fading channels with AWGN. For a given set of symbols transmitted by the $K$ CRUs as well as their FH addresses, the number of CRUs activating the $(m, l)$ th TF element is then known, which is expressed as $K_{m l}, 0 \leq$ $K_{m l} \leq K$. Then, the decision variable $R_{m l}$ is

$R_{m l}=\left(\mathfrak{R}\left\{\sum_{k=1}^{K_{m l}} h_{m}^{(k)}+n_{m l}\right\}\right)^{2}+\left(\mathfrak{I}\left\{\sum_{k=1}^{K_{m l}} h_{m}^{(k)}+n_{m l}\right\}\right)$

When communicating over flat Rayleigh fading channels, both the real and imaginary parts of $h_{m}^{(k)}$ obey the Gaussian distributions with zero mean and a variance of $1 / 2$. Hence, both the real and imaginary parts of (11) are Gaussian random variables with zero mean and a variance of $K_{m l} / 2+\sigma^{2} / 2$. Therefore, $R_{m l}$ obeys the central $\chi^{2}$-distribution with two degrees of freedom with the PDF

$$
\begin{aligned}
f\left(R_{m l} \mid \boldsymbol{s},\left\{\boldsymbol{a}_{k}\right\}\right) & =\frac{1}{K_{m l}+\sigma^{2}} \exp \left(-\frac{R_{m l}}{K_{m l}+\sigma^{2}}\right) \\
m & =0,1, \ldots, Q-1 ; \quad l=0,1, \ldots, L-1
\end{aligned}
$$

Upon substituting (12) to (10), the optimum noncoherent MLMUD can now be stated as

$$
\begin{aligned}
\hat{\boldsymbol{s}} & =\arg \max _{\left\{X_{k} \in \mathcal{X}\right\}_{k=1}^{K}}\left\{\prod_{m=0}^{Q-1} \prod_{l=0}^{L-1} \frac{1}{K_{m l}+\sigma^{2}} \exp \left(-\frac{R_{m l}}{K_{m l}+\sigma^{2}}\right)\right\} \\
& \triangleq \arg \min _{\left\{X_{k} \in \mathcal{X}\right\}_{k=1}^{K}}\left\{\sum_{m=0}^{Q-1} \sum_{l=0}^{L-1}\left[\ln \left(K_{m l}+\sigma^{2}\right)+\frac{R_{m l}}{K_{m l}+\sigma^{2}}\right]\right\}
\end{aligned}
$$

The search space's size for the optimum noncoherent MLMUD, as shown in (13), is $M^{K}$, which leads to high complexity even for a moderate number of CRUs supported.
In order to reduce the detection complexity, the detection may be based on the hard-decision TF matrix as shown in Fig.4(b). Furthermore, some posterior noncoherent interference cancellation (IC) techniques, such as minimum-distance decoding IC and iterative posterior IC, etc. [12,14-16], may be invoked for improving the detection performance. However, these algorithms become less efficient, when communicating over fading channels with AWGN [12]. Furthermore, selecting a near-optimum threshold for deriving the hard-decision TF matrix in the form of Fig. 4(b) is usually very hard in practice.

Considering the above issues, in this paper, we invoke a soft-decision based suboptimum noncoherent ML-MUD, the principles of which are as follows. First, the receiver knows that there are at most $K$ elements activated by the $K$ CRUs in each column of the TF matrix $R$ shown in Fig. 4(a). Hence, the detector only needs to test these $K$ symbols. However, due to the fading and AWGN, which result in false-alarms, we select $[\gamma K], 1 \leq \gamma \leq Q / K$, elements from each column of $R$, rendering them the possible candidates activated by the $K$ CRUs. Second, an accompanying TF matrix $\boldsymbol{R}_{a}$ as shown in Fig. 4(c) is constructed based on these candidate elements. Specifically, $\boldsymbol{R}_{a}$ is constructed according to the following policy: the $(m, l)$ th element is ' 1 ' (or 'marked') if the corresponding element in $R$ of Fig. 4(a) is a candidate element; otherwise, the $(m, l)$ th element is ' 0 ' (or 'empty'). Third, the accompanying TF matrix $\boldsymbol{R}_{a}$ is respectively dehopped by the FH addresses of the $K$ CRUs, yielding $K$ dehopped matrices expressed as $\boldsymbol{D}_{1}, \boldsymbol{D}_{2}, \ldots, \boldsymbol{D}_{K}$, in the form of Fig. 4(d), (e), (f) and (g) for the example considered. Forth, based on the de-hopped matrices $D_{1}, D_{2}, \ldots, D_{K}$, the possible symbols transmitted by the $K$ CRUs can be initially identified. Specifically, depended on the affordable detection complexity, in $\boldsymbol{D}_{k}$, the rows with the number of marked elements no less than $(L-\varepsilon)$ are identified and mapped to the MFSK symbols, which are treated as the possible symbols transmitted by the $k$ th CRU. Here, $\varepsilon$ is a preset integer. For example, in Fig. 4(d), (e), (f) and (g), the possible transmitted symbols for $k=1,2,3,4$ can be identified as $\left\{X_{1} \in\{0,2\} ; X_{2} \in\{3\} ; X_{3} \in\{0\} ; X_{4} \in\{1\}\right\}$, when setting $\varepsilon=0$. Let the possible symbols transmitted by the $K$ CRUs be collected in $\left\{X_{i} \in \mathcal{X}_{i}\right\}_{i=1}^{K}$. Fifth, having obtained the set $\left\{X_{i} \in \mathcal{X}_{i}\right\}_{i=1}^{K}$ for the possible symbols transmitted, the receiver re-encodes these possible symbols using the $K$ CRUs' FH addresses to form a TF matrix $\boldsymbol{R}_{t}$ having the same form as $\boldsymbol{R}_{a}$. The marked elements in $\boldsymbol{R}_{t}$ are collected in a set $\mathcal{M}$. Finally, based on the set $\left\{X_{i} \in \mathcal{X}_{i}\right\}_{i=1}^{K}$ of the candidate symbols and the set $\mathcal{M}$ containing the elements possibly activated, the suboptimum ML-MUD detects $s$ according to

$$
\hat{\boldsymbol{s}}=\arg \min _{\left\{X_{k} \in \mathcal{X}_{k}\right\}_{k=1}^{K}}\{\underbrace{\sum_{m=0}^{Q-1} \sum_{l=0}^{L-1}}_{(m, l) \in \mathcal{M}}\left[\ln \left(K_{m l}+\sigma^{2}\right)+\frac{R_{m l}}{K_{m l}+\sigma^{2}}\right]\}
$$

Note that, in (14), we only need to consider the elements falling within $(m, l) \in \mathcal{M}$. This is because, for the other 
elements not included in $\mathcal{M}$, we have $K_{m l}=0$, implying that these elements are not activated by any CRUs. Hence, their corresponding terms are common to the optimization and can be eliminated without affecting the solution.

It can be seen from (14) that the search space $\left\{X_{k} \in\right.$ $\left.\mathcal{X}_{k}\right\}_{k=1}^{K}$ for the suboptimum ML-MUD can be significantly smaller than that of the optimum ML-MUD stated in (13). Specifically for the example considered in Fig. 4, there are only two candidate symbol vectors to consider, in contrast to $M^{K}=8^{4}=4096$ candidate vectors for the optimum ML-MUD. The first candidate symbol vector is $\left\{X_{1}, X_{2}, X_{3}, X_{4}\right\}=\{0,3,0,1\}$ and the second is $\left\{X_{1}, X_{2}, X_{3}, X_{4}\right\}=\{2,3,0,1\}$. Therefore, the suboptimum noncoherent ML-MUD may require significantly lower implementation complexity than the optimum ML-MUD.

\section{Simulation Results}

In our simulations, we assume that the arrival rate of the PUs is $\lambda=1$ and that the average occupying time of a frequency band by a PU $1 / \mu=1 / 2$, resulting in $\lambda / \mu=1 / 2$. The number of frequency bands for operation of the CR system is given by $Q=\bar{C}$, which experience independent identically distributed (iid) Rayleigh fading. For simulation of the BER, we assume that $M \leq \bar{C} \leq C$, implying that the CR system is active. By contrast, for simulation of the throughput performance, we assume that $0 \leq \bar{C} \leq C$. Furthermore, for implementation of the suboptimum ML-MUD, we set the parameter $\varepsilon=1$, meaning that the rows with $L$ and $L-1$ entries are considered by the suboptimum ML-MUD. Additionally, random FH addresses are assumed.

Fig. 5 shows the BER performance of the dynamic FFH/MFSK system employing the suboptimum ML-MUD, when communicating over Rayleigh fading channels. The parameters for the primary radio system were $C=128, K_{P}=$ $8, C_{P}=16$. For the CR system, in Fig. 5(a), $M=16$ was assumed, while in Fig. 5(b) the value of $M$ was $M=$ $2^{i n t\left(\log _{2} \bar{C}\right)}$, depending on the value of $\bar{C}$. Furthermore, the number of entries in each column of $R$ chosen for forming $\boldsymbol{R}_{a}$ in order to find the candidate symbols was $2 K$. From the results of Figs. 5(a) and (b), we observe that the suboptimum ML-MUD is a high-efficiency detection scheme. There are no error-floors observed, even the system supports $K>L$ number of CRUs. At a given SNR per bit, the BER only slightly increases, as the number of CRUs increases. Specifically, at the BER of $10^{-3}$, in Fig. 5(a), an extra of about $5 \mathrm{~dB}$ of SNR is required, in order for the CR system to support 6 CRUs, in comparison with the $\mathrm{CR}$ system supporting $K=1 \mathrm{CRU}$ and using the optimum ML detector. By contrast, as shown in Fig. 5(b), about $7 \mathrm{~dB}$ of extra SNR is required, in order for the CR system to support $6 \mathrm{CRUs}$, in comparison with the CR system supporting $K=1 \mathrm{CRU}$ and using the optimum ML detector. The reason for the increased SNR in Fig. 5(b) in comparison with that in Fig. 5(a) is because, in Fig. 5(a) $M=16$ is fixed, while in Fig. 5(b) the value of $M$ is dynamic, which provides a higher throughput than the system considered in Fig. 5(a).

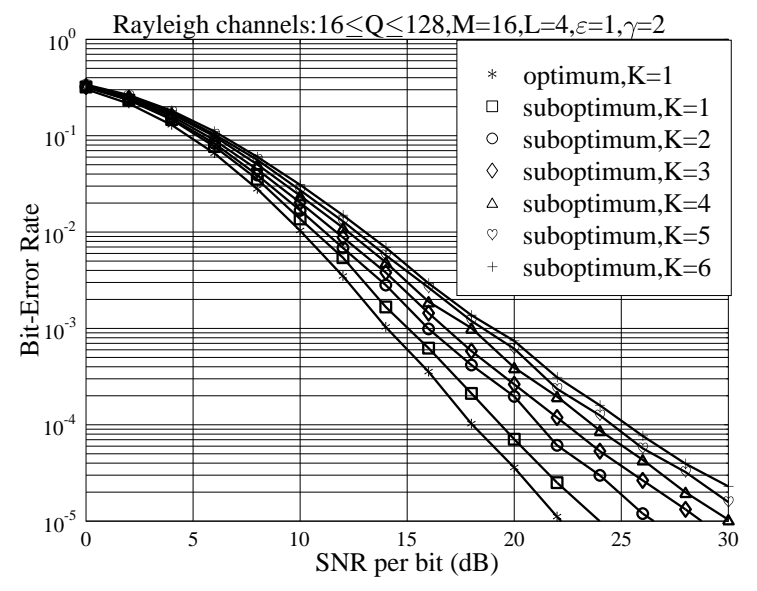

(a) $M=16$

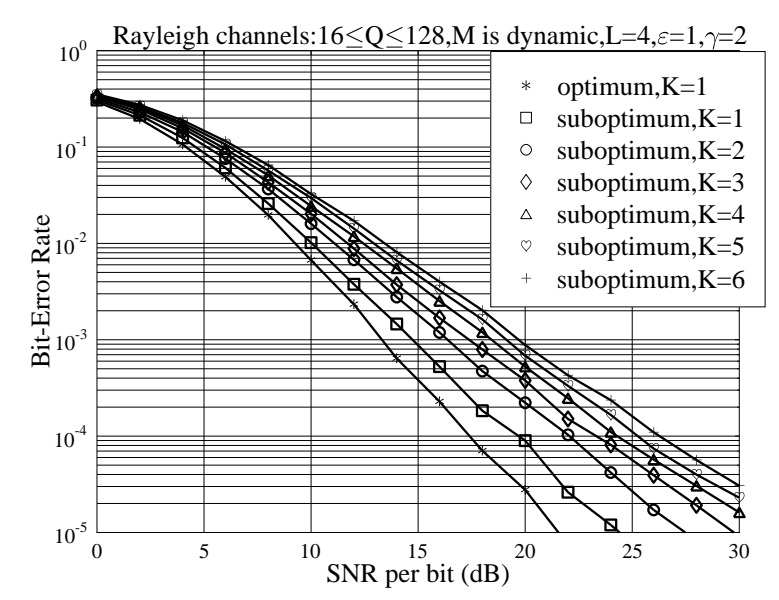

(b) Dynamic $M$

Fig. 5. BER versus average SNR per bit performance of the dynamic FFH/MFSK system employing suboptimum noncoherent ML-MUD, when communicating over Rayleigh fading channels.

Fig. 6 illustrate the throughput achievable by the FFH/MFSK systems. The throughput depicted were found as follows. Given the values of $Q=\bar{C}, M$ and the BER required, the maximum number of CRUs supportable by the dynamic FFH/MFSK system can be determined, say it is $K$. The throughput is then calculated as $K \times \log _{2} M$. From the results of Fig. 6, we have the following observations. First, a FFH/MFSK system using dynamic MFSK is capable of achieving higher throughput than that using fixed MFSK. Second, for a given BER demanded, the throughput increases sharply, as the SNR increases, especially, when the demanded BER is relatively low. Third, when we compare Fig. 6(a) with Fig. 6(b), we can see that, for a given required BER and a given but reasonable SNR per bit, the FFH/MFSK CR system using $C=256$ is capable of providing a higher throughput than the FFH/MFSK CR system using $C=128$. This observation implies that, although the PUs' arrival rate is 


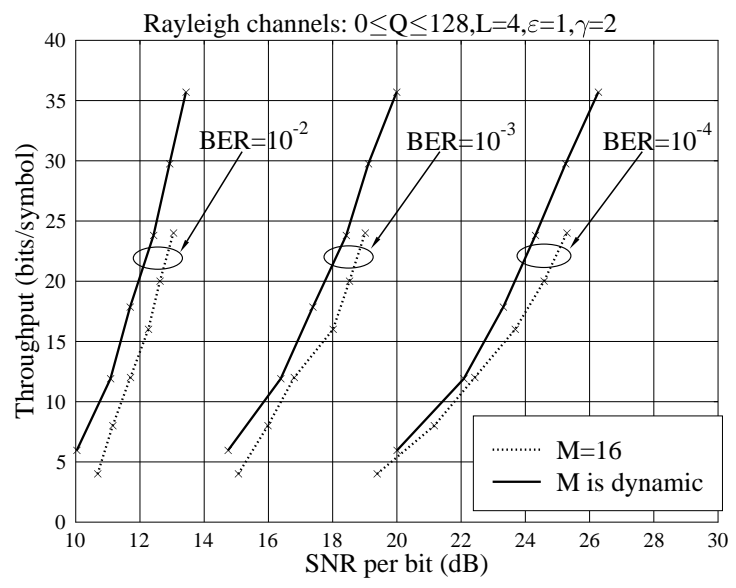

(a) $C=128, L=4$

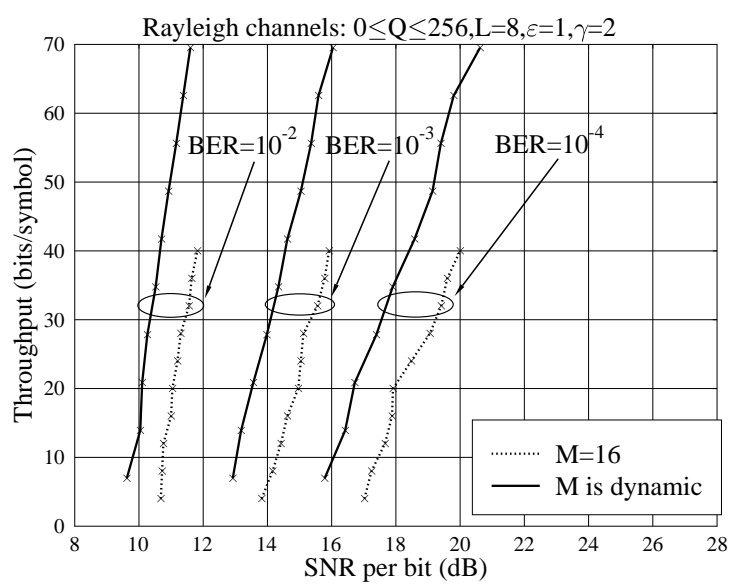

(b) $C=256, L=8$

Fig. 6. Throughput versus average SNR per bit performance of the FFH/MFSK system employing suboptimum ML-MUD, when communicating over Rayleigh fading channels.

the same, increasing the total number of frequency bands of $C$ results in that the FFH/MFSK CR system can obtain in average a higher number of frequency bands for communications, yielding lower BER or higher throughput.

\section{CONCLusions}

In this paper, we have proposed a scheme of FFH/MFSK for CRUs to access dynamically the available frequency spectrums in the CR environments, where PUs activate their communication according to a Poisson process. The error and throughput performance of the dynamic FFH/MFSK systems employing suboptimum noncoherent ML-MUD have been investigated by simulations, when assuming communications over Rayleigh fading channels. Our studies and performance results show that our proposed dynamic FFH/MFSK constitutes a highly promising DMA scheme for application in CRs. It is capable of achieving a promising throughput with reasonable error performance. Owing to the employment of noncoherent
FFH/MFSK and noncoherent MUD techniques, the proposed DMA scheme is low-complexity and high-flexibility. It can implement seamless and smooth handoff between different frequency bands, when the available spectrum bands change. Furthermore, it requires only simple spectrum control and spectrum allocation protocols.

\section{ACKNOWLEDGEMENT}

The work of the first and third authors was supported by the National Basic Research Program of China (973 Program, Grant No. 2010CB731803).

\section{REFERENCES}

[1] FCC, Spectrum Policy Task Force Report, ET Docket No. 02-155, November 2002.

[2] FCC. ET Docket No. 03-322. Notice of Proposed Rule Making and Order, December 2003.

[3] J. Mitola, "Cognitive radio for flexible mobile multimedia communications," Mobile Networks and Applications, vol. 6, no. 5, pp. 435 - 441, 2004.

[4] I. F. Akyildiz and et.al, "NeXt generation/dynamic spectrum access/cognitive radio wireless networks: A survey," Computer Networks, vol. 50, no. 13, pp. 2127 - 2159, 2006.

[5] T. Yucek and H. Arslan, "A survey of spectrum sensing algorithms for cognitive radio applications," IEEE Communication Surveys \& Tutorials, vol. 11 , no. 1 , pp. 116 - 130, 2009.

[6] I. F. Akyildiz and Y. Li, "OCRA: OFDM-based cognitive radio networks," Broadband and Wireless Networking Laboratory Technical Report, March 2006.

[7] T. Weiss, J. Hillenbrand, A. Krohn and F. K. Jondral, "Mutual interference in OFDM based spectrum pooling systems," IEEE Vehicular Technology Conference, vol. 4, pp. 1873 - 1877, May 2004.

[8] T. Weiss and F. Jondral, "Spectrum pooling: An innovative strategy for enhancement of spectrum efficiency," IEEE Communications Magazine, vol. 42, no. 3, pp. 8 - 14, March 2004.

[9] D. Kendall, "Stochastic processes occurring in the theory of queues and their analysis by the method of the imbedded Markov chain," The Annals of Mathematical Statistics, vol. 24, no. 3, pp. 338 - 354, 1953.

[10] A. Goldsmith and et.al, "Breaking spectrum gridlock with cognitive radios: an information theoretic perspective," Proceedings of the IEEE, vol. 97, no. 5, pp. 894 - 914, May 2009.

[11] D. Bertsekas and R. Gallager, Data Networks (2nd Ed.). Prentice Hall, Upper Saddle River, New Jersey, USA, 1992.

[12] L.-L. Yang, Multicarrier Communications. Chichester, United Kingdom: John Wiley, 2009.

[13] J. G. Proakis, Digital Communications (3rd Ed.). McGraw Hill, 1995.

[14] T. Mabuchi, R. Kohno and H. Imai, "Multiuser detection scheme based on canceling cochannel interference for MFSK/FH-SSMA system," IEEE Journal on Selected Areas in Communications, vol. 12, no. 4, pp. 593 604, May 1994.

[15] U. Fiebig, "Iterative interference cancellation for FFH/MFSK MA systems," IEE Proceedings Communications, vol. 143, pp. 380 - 388, December 1996.

[16] K. W. Halford and M. Brandt-Pearce, "Multistage multiuser detection for FHMA," IEEE Transactions on Communications, vol. 48, no. 9, pp. 1550 - 1562, September 2000. 